\title{
A novel SLC6A8 mutation associated with intellectual disabilities in a Chinese family exhibiting creatine transporter deficiency: case report
}

\author{
Qin Wang, Jingxin Yang, Yang Liu, Xingping Li, Fuwei Luo and Jiansheng Xie (D)
}

\begin{abstract}
Background: X-linked creatine transporter deficiency (OMIM\#300036,CRTR-D) is characterized by cerebral creatine deficiency, intellectual disabilities, severe speech impairment, seizures and behavioral problems. Mutations in the creatine transporter gene SLC6A8, a member of the solute-carrier family 6 mapped to Xq28, have been reported to cause the creatine transporter deficiency.

Case presentation: The proband presented at 5 yrs. 1 month of age with delays in intellectual and development, seizures and behavioral problems. A novel missense mutation, c.1181C > A (p.Thr394Lys), in the SLC6A8 gene (NM_005629.3) was detected via targeted exome sequencing, and then validated by Sanger sequencing. Multiple in silico variant effect analysis methods, including SIFT, PolyPhen2, PROVEAN, and Mutation Taster predicted that this variant was likely damaging or diseasing-causing. This hemizygous variation was also identified in the affected brother with the same clinical condition and inherited from the heterozygous carrier mother. The diagnosis was suggested by increased urinary creatine/creatinine ( $\mathrm{Cr}: \mathrm{Crn}$ ) ratio and markedly reduced creatine content peak by brain proton magnetic resonance spectroscopy (MRS). The proband's mother became pregnant with a 3rd sibling, in whom the Sanger sequencing result of c.1181C > A was negative.

Conclusion: The novel mutation C.1181C > A in the SLC6A8 gene reported in a Chinese family has expanded the mutation spectrum of CRTR-D. The combination of powerful new technologies such as targeted exome sequencing with thorough systematic clinical evaluation of patients will improve the diagnostic yield, and assist in genetic counselling and prenatal diagnosis for suspected genetic disorders.
\end{abstract}

Keywords: Creatine transporter deficiency (CRTR-D), X-linked, Intellectual disabilities, SLC6A8, Targeted exome sequencing

\section{Background}

Creatine is essential in energy metabolism and plays a vital role in high-energy requirement organs such as muscle, brain and heart, as it facilitates the resynthesis of ATP from ADP through phosphocreatine $[1,2]$. In humans, about half of the creatine is synthesized in the kidney and liver, and the other half is obtained by dietary intake. Although the brain is capable of producing minor amounts of creatine, the major proportion is taken up from the blood via solute

\footnotetext{
* Correspondence: jsxieszmch@163.com

Shenzhen Maternity and Child Healthcare Hospital, No. 3012, Fuqiang Road Shenzhen 518028, Guangdong, China
}

carrier family 6 (neurotransmitter transporter, creatine), member 8 (SLC6A8) [2, 3]. Pathological mutations in $S L C 6 A 8$ affect the creatine transport into the brain and muscle. Creatine transporter deficiency (CRTR-D) (OMIM 300036) is the most common cerebral creatine deficiency syndrome (CCDS) and may be one of the leading causes of $\mathrm{X}$-linked mental retardation (X-LMR) in males [4-6]. Clinical symptoms of creatine transporter deficiency in affected hemizygous males include intellectual disability (ID), development delay, expressive speech, and language delay, epilepsy, and autistic behavior [7-9]. Female carriers may exhibit mild cognitive impairment with behavior and 
learning problems [10]. The diagnosis of creatine transporter deficiency is based on clinical presentation and/or increased urine creatine/creatinine ratio, abnormal brain magnetic resonance spectroscopy and reduced brain creatine content. DNA analysis of disease-causing mutations in SLC6A8 or measurement of impaired creatine uptake in fibroblasts are confirmatory of the clinical diagnosis $[11,12]$.

The creatine transporter gene, SLC6A8, also known as CT1 or CRTR, is a member of the solute-carrier family 6 mapped to Xq28. The gene is expressed in most tissues and the creatine transporter (CT1) encoded by this gene is 635 amino acids long with 12 transmembrane domains and is required for the uptake of creatine in muscles and brain [6]. Defects in SLC6A8 can result in X-linked creatine deficiency syndrome. The prevalence of SLC6A8 deficiency is estimated at $0.8 \%$ to $2.1 \%[5,13]$. Since the first report of creatine transporter deficiency in 2001, many novel variants have been detected. The online database collecting the SLC6A8 variants has been established by Dr. GS Salomons (http://www.LOVD.nl/SLC6A8), which includes updated clinical and genetical data [14]. Currently, more than 140 SLC6A8 variants have been published.

Exome sequencing has become the most widely used targeting exome sequencing method, especially for monogenic (Mendelian) diseases [15]. Since exonic mutations cause the majority of monogenic diseases, the application of targeted exome panel sequencing has largely contributed to the identification of new disease-causing genes and confirmed its advantages in reducing the diagnostic odyssey for many suspect genetic disorders [16, 17]. In China, few CRTR-D patients have been reported. In this study, we present the detection of a novel mutation c.1181C > A (p.Thr394Lys) in the SLC6A8 gene via targeted exome sequencing in a Chinese family with CRTR-D. The mutation was confirmed by Sanger sequencing. The detection of novel pathogenic variants in the exome by targeted exome sequencing has been a powerful and effective diagnostic tool in determining the molecular basis of genetic disorders.

\section{Case presentation}

A male patient aged 5-year-and-1-month was referred for genetic evaluation of development and speech delay, intellectual disabilities at the genetic counselling clinic in Shenzhen Maternal and Child Healthcare Hospital. The parents described that an affected brother also presented the same clinical phenotype but was not available for the clinical examination. The chromosome karyotype and chromosomal microarray analysis (CMA) in the proband were normal. The mother was pregnant again and pursued genetic counseling. The proband was subject to comprehensive neurological testing including the Gesell Developmental index. Molecular genetic tests and biochemical and neurochemical analysis were performed on the proband. The present study was approved by the hospital's Institutional Review Board and written informed consent was obtained from their parents.

\section{Clinical findings}

The proband was the second boy of healthy nonconsanguineous parents (pedigree in Fig. 1a). He was born at
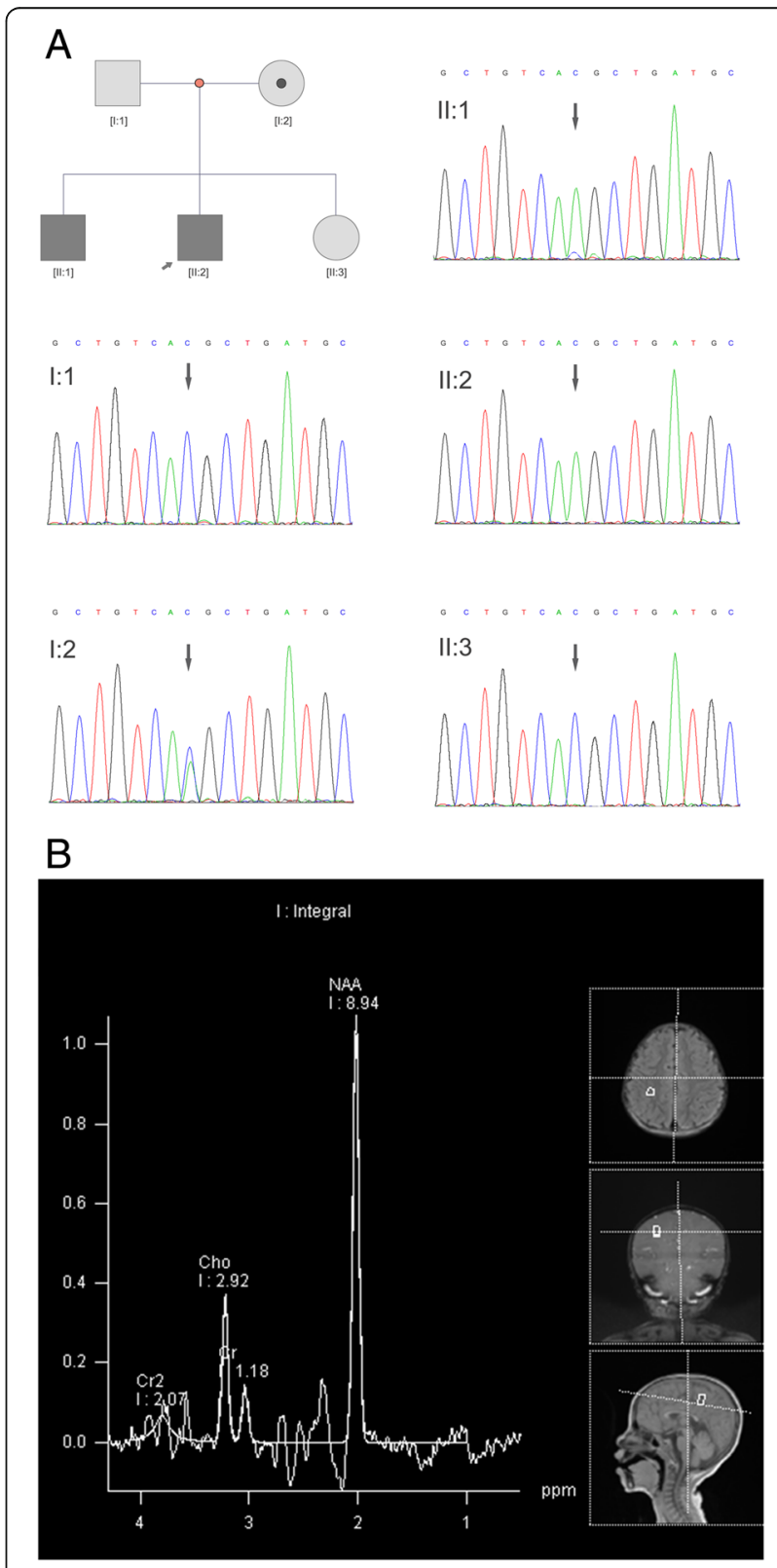

Fig. 1 Molecular genetic tests and neurochemical analysis results in the index patient. a. Family tree of the proband and Sanger sequencing results in this study. Sanger sequencing validated the mutation, c.1181C > A (p.Thr394Lys), in the SLC6A8 gene in family members as $1: 1$ wildtype, I:2 heterozygous carrier, II:1 hemizygous, $\|: 2$ hemizygous and II:3 wildtype. b. Proton magnetic resonance spectroscopy (MRS), examination using a 3.0-T system on the brain showed marked reduction of the brain creatine peak (left part), and brain MRI showed a thin corpus callosum in the proband (right part) 
39 weeks of gestation from an uneventful pregnancy and delivered by Caesarean section (weight, 3600 g; length, $50 \mathrm{~cm}$; head circumference, $36 \mathrm{~cm}$ ). He showed head control at 12 months, ability to sit by himself at 15 months, and walking with aid at 20 months. His verbal language was nearly absent and he made no visual contact. He suffered from seizures from 6 months old. He had no craniofacial dysmorphism. Gastrointestinal problems such as chronic constipation or nausea were noted in the proband. The physical examination on the proband showed $95 \mathrm{~cm}$ height, weight $18.2 \mathrm{~kg}$ and developmental and language delay. The proband also had an electroencephalogram (EEG) test, which showed sharp and slow waves in sleep during 24-h EEG monitoring. A brain stem auditory-evoked potential (BAEP) test showed mild abnormality. The proband had a Children's Autism Rating Scale (CARS) score of 33, which indicated mildly autistic characteristics. The Gesell developmental scale test was used to evaluate the proband. Both the development age (DA) and developmental quotient (DQ) data showed extremely low grades which suggested severe development delay (adaptability, $\mathrm{DA}=14.23 \mathrm{mo} ., \mathrm{DQ}=23$; gross motor, $\mathrm{DA}=$ $26.37 \mathrm{mo}$., $\mathrm{DQ}=43$; fine movement $\mathrm{DA}=15.87 \mathrm{mo}$., $\mathrm{DQ}=$ 26 ; vocabulary $\mathrm{DA}=13.07 \mathrm{mo}$., $\mathrm{DQ}=21$; personal-social skill $\mathrm{DA}=13.3 \mathrm{mo}$., $\mathrm{DQ}=22$ ). The test results are depicted in Additional file 1: Figure S1A. The affected brother of the proband (II:1) was not available for the physical examination. The parental description of the clinical phenotype of the brother was mostly the same as the proband. The parents were physically healthy and indicated no significant past medical, surgical or family history.

\section{Methods and results}

DNA samples were provided from the index patient and other family members, which were extracted as previously described [18]. The present study used the TruSight One Sequencing Panel and NextSeq 500/550 Mid Output v2 kit (300 cycles) with high depth of coverage for 4813 target genes (approximately 62000 target exons) that are associated with clinically relevant phenotypes. An average sequencing depth of $136.88 \mathrm{x}$ was achieved and $98.25 \%$ of targeted variants were covered at least to a 10x depth, and $97.04 \%$ of targeted variants were covered at least by $20 x$. The total detected variants numbered 24594, which included 21,733 SNPs, 1,182 insertions and 1,679 deletions respectively. The data were analyzed on the TGex (Translational Genomics Expert) platform featuring with the VarElect scoring system [19]. A missense mutation, c.1181C > A (p.Thr394Lys), in the SLC6A8 gene was called with high probability as a candidate mutation.

Sanger sequencing was performed to confirm the SLC6A8 gene c.1181C >A mutation (forward primer 5' ACGG AACTTGTCAGATTGT3', and reverse primer 5'CAAC AGCATGAAGAAGAACA3'). The father (I:1) was wildtype and the mother (I:2) was heterozygous for the c.1181C > A variation. The affected brother (II:1) and the proband (II:2) both carried the hemizygous variation of c.1181C $>$ A. The pregnant mother had an amniocentesis at 22 weeks and Sanger sequencing targeting the SLC6A8 gene c. $1181 \mathrm{C}>\mathrm{A}$ was performed. The result showed a wild-type allele (II:3) and the mother gave birth to a healthy baby girl (Fig. 1a). In silico variant prediction analysis methods, including SIFT, PolyPhen2, PROVEAN, and Mutation Taster demonstrated this variant had probably damaging or diseasing-causing effects.

\section{Biochemical and neurochemical analysis}

Biochemical screening was performed with blood and urine samples from the proband and his mother. The creatine/creatinine (Cr:Crn) ratio was determined by liquid chromatography-mass spectrometry with deuterated internal standards in two urine samples taken on different days. A urine creatine test of the proband showed significantly elevated levels of creatine $(0.805 \mathrm{mg} / \mathrm{ml}$, normal control $0.160 \pm 0.177 \mathrm{mg} / \mathrm{ml}$ ) (Additional file 1: Figure S1B), and the creatine/creatinine ratio was significantly elevated compared to controls. Proton magnetic resonance spectroscopy (MRS, Magnetom Skyra 3.0-T, Siemens Healthcare $\mathrm{GmbH}$, Erlangen, Germany), examination using a 3.0-T system at the brain left parietal lobe, right parietal lobe and genu of corpus callosum all showed marked reduction of the brain creatine peak (Fig. 1b left part). Brain MRI showed a thin corpus callosum in the proband (Fig. 1b right part). The MRS and MRI examination of the mother (I:2) showed normal results (Additional file 1: Figure S1C).

\section{Discussion}

In the present study, targeted exome sequencing revealed a novel missense mutation, c.1181C > A (p.Thr394Lys), in the SLC6A8 gene (NM_005629.3), resulting in CRTR deficiency syndrome in a Chinese family. The index patient was referred for unexplained intellectual disability, with severe speech delay, seizures and autistic behaviors. High-throughput sequencing was performed on the proband, and the results revealed a pathogenic missense mutation in the creatine transport gene SLC6A8. The missense variation c. $1181 \mathrm{C}>\mathrm{A}$ was in the predicted transmembrane domain 8 and in silico analysis predicted this variant as possibly damaging. The diagnosis of CRTR-D was confirmed by the urinary creatine/creatinine measurement and MRS of the brain. The hemizygous index male patient presented with the characteristic phenotype comprising intellectual disability, severe speech delay, epilepsy, and behavioral disturbances. The hemizygous brother was identified through candidate variant analysis, and his clinical features were identical to the proband, according to the parental reports. Gastrointestinal problems such as vomiting and constipation were also noted in both brothers. 
Neither brother had obvious craniofacial dysmorphism. The clinical presentations are similar to the previous clinical reports of CRTR-D patients [20]. The proband's heterogenous mother was physically normal. The proband's mother became pregnant with a 3rd sibling, on whom amniocentesis and Sanger sequencing result of c.1181C > A was negative.

CRTR-D is reported to be the most frequent disorder of creatine synthesis and cellular transport and is supposed to be one of the more common causes of X-linked mental retardation (XLMR) [5, 20]. The diagnosis of CRTR-D includes the clinical presentation of mental retardation (MR), expressive speech and language delay, epilepsy, developmental delay and autistic behavior in affected males. Laboratory hallmarks include an increased urinary creatine/creatinine ( $\mathrm{Cr}: \mathrm{Crn})$ ratio, which is a widely available test. A reduction of the creatine signal in the proton magnetic resonance spectroscopy (H-MRS) of the brain is also a very sensitive screening method, although H-MRS is not regularly equipped in many centers. Additional molecular genetic testing in the form of the sequencing of the SLC6A8 gene is necessary to confirm the diagnosis. Next generation sequencing will play more roles in identifying the genetic causes of CRTR-D. The creatine uptake test in cultured fibroblasts should be studied to prove the pathogenicity in case of a novel unclassified variant. The estimated frequency of SLC6A8 deficiency in males with XLMR is $2.1 \%, 1.5 \%$ and $0.8 \%$ in different studies. The prevalence in family cases is $5.4 \%$ $[5,13,21]$. The estimated carrier frequency of CRTR-D is $0.024 \%$ in females in the general population [22]. A total of $142 S L C 6 A 8$ variants have been reported in 667 individuals (according to the LOVD SLC6A8 database), which mainly in Western populations. All known mutations are either de novo (30\%), or maternally inherited (70\%) [4]. Phenotypic variability has been observed in male patients, from mild to severe clinical symptoms, which may depend on the location of the missense mutation in the protein structure $[7,8,23,24]$. In our study, the two siblings had the same hemizygous mutation, which was maternally inherited. They were severely affected, and clinical follow-up should be done as previous reports document progressive cerebral atrophy and the broader clinical presentation of SLC6A8 deficiency in reported adult patients $[25,26]$. Since the diagnostic guidelines of CRTR-D have been well established, more patients should be recruited to study the genotype-phenotype correlation and mutation spectrum in Chinese for its high frequency in patients with unexplained mental retardation.

Currently CRTR-D is an untreatable disease and the pathogenic mechanism is not entirely clear. Recent evidence proposed the new role of creatine as neuromodulator or neurotransmitter based on the prediction that cerebral creatine deficiency in CRTR-D derives from a failure of creatine recycling following release [27]. Creatine depletion in the brain may reduce the white matter brain volume and thus affect the cognitive ability [28]. A mouse model of CRTR-D has revealed that Cr deficiency in the cerebral cortex and hippocampus regions is apparent in the cognitive defects. Endogenous uptake of creatine does not compensate for the loss of creatine in the mouse or human brain $[29,30]$. RNA sequencing has suggested that SLC6A8 mutation will result in gene expression alterations, especially genes encoding components of the extracellular matrix or cell structure. Dysfunctional extracellular matrix molecules in SLC6A8-deficient neurons are related to neurodegenerative disorders, learning and epilepsy [31]. The restoration of the extracellular matrix could become a therapeutic breakthrough for CRTR-D.

\section{Conclusion}

In conclusion, a novel missense mutation, c.1181C > A (p.Thr394Lys), in the SLC6A8 gene was detected in a Chinese family through targeted exome sequencing. The diagnosis of CRTR-D was confirmed by urinary creatine/ creatinine measurement as well as MRS of the brain. The combination of targeted exome sequencing with systematic clinical evaluation of patients used in suspected genetic disorders may improve diagnostic yield, assist in the medical care of patients and offer genetic counseling and prenatal diagnosis for family members.

\section{Additional file}

Additional file 1: Figure S1. A. Gesell developmental scale evaluated the proband as severely developmentally delayed. B. Urine creatine was significantly increased $(0.805 \mathrm{mg} / \mathrm{ml}$ ) in the proband (peak height 25,000, upper left) compared with the normal control (NC) value of $0.160 \pm$ $0.177 \mathrm{mg} / \mathrm{ml}$ (peak height 3,200, below left). The internal standard peak is the reference for the test at right. C. Proton magnetic resonance spectroscopy (MRS), examination using a 3.0-T system on the brain showed normal brain creatine peak (left part) and brain MRI showed normal corpus callosum in the proband's mother (right part). (TIF $5633 \mathrm{~kb}$ )

\section{Abbreviations}

CCDS: Cerebral creatine deficiency syndrome; $\mathrm{Cr}: C r n$ : Creatine/creatinine; CRTR-D: Creatine transporter deficiency; DA: Development age;

DQ: Developmental quotients; H-MRS: Proton magnetic resonance spectroscopy; ID: Intellectual disability; MRI: Magnetic resonance imaging; SLC6A8: Solute Carrier Family 6 (Neurotransmitter Transporter, Creatine), Member 8; X-LMR: X-Linked mental retardation

\section{Acknowledgements}

The authors appreciate the family to taking part in this study and for permission to publish the report. We would like to thank Dr. Zhang Gongwei for his technical support.

\section{Funding}

This work was supported by Shenzhen Health and Family Planning Commission Research Project (SZFZ2018047), Shenzhen Science and Technology Innovation Committee (Grant Number JCYJ20170413092818116).

Availability of data and materials

The datasets generated during and/or analyzed during the current study are available from the corresponding author on reasonable request. 


\section{Authors' contributions}

WQ, performed targeted exome sequencing, data analysis, literature review and drafted the manuscript; YJ/LY/LX, performed Sanger sequencing analysis and patient record management; LF, performed chromosome analysis; XJ, organized this study, reviewed clinical and laboratory data, and finalized this manuscript. All authors read and approved the final manuscript.

\section{Ethics approval and consent to participate}

A statement on ethics approval and consent for this study was approved by the Institutional Ethics Review Board, Shenzhen Maternity and Child Healthcare Hospital. Written informed consent was obtained from the parents to take part in this study. A copy of the written consent is available for review by the Editor of this journal.

\section{Consent for publication}

Written informed consents for publication of their clinical details and/or clinical images were obtained from the patient or parents.

\section{Competing interests}

The authors declare that they have no competing interests.

\section{Publisher's Note}

Springer Nature remains neutral with regard to jurisdictional claims in published maps and institutional affiliations.

Received: 26 July 2018 Accepted: 22 October 2018

Published online: 06 November 2018

\section{References}

1. Wyss M, Kaddurah-Daouk R. Creatine and creatinine metabolism. Physiol Rev. 2000:80(3):1107-213.

2. Joncquel-Chevalier Curt M, Voicu PM, Fontaine M, Dessein AF, Porchet N, Mention-Mulliez K, et al. Creatine biosynthesis and transport in health and disease. Biochimie. 2015;119:146-65

3. Brosnan JT, Brosnan ME. Creatine: endogenous metabolite, dietary, and therapeutic supplement. Annu Rev Nutr. 2007;27:241-61.

4. van de Kamp JM, Mancini GM, Salomons GS. X-linked creatine transporter deficiency: clinical aspects and pathophysiology. J Inherit Metab Dis. 2014; 37(5):715-33.

5. Rosenberg EH, Almeida LS, Kleefstra T, deGrauw RS, Yntema HG, Bahi N et al. High prevalence of SLC6A8 deficiency in X-linked mental retardation. Am J Hum Genet. 2004:75(1):97-105.

6. Salomons GS, van Dooren SJ, Verhoeven NM, Cecil KM, Ball WS, Degrauw TJ, et al. X-linked creatine-transporter gene (SLC6A8) defect: a new creatinedeficiency syndrome. Am J Hum Genet. 2001;68(6):1497-500.

7. Puusepp H, Kall K, Salomons GS, Talvik I, Männamaa M, Rein R, et al. The screening of SLC6A8 deficiency among Estonian families with X-linked mental retardation. J Inherit Metab Dis. 2009:33(S3):5-11.

8. Garcia P, Rodrigues F, Valongo C, Salomons GS, Diogo L. Phenotypic variability in a portuguese family with $\mathrm{x}$-linked creatine transport deficiency. Pediatr Neurol. 2012;46(1):39-41.

9. van de Kamp JM, Betsalel OT, Mercimek-Mahmutoglu S, Abulhoul L, Grunewald S, Anselm I, et al. Phenotype and genotype in 101 males with X-linked creatine transporter deficiency. J Med Genet. 2013;50(7):463-72.

10. Stockler S, Schutz PW, Salomons GS. Cerebral creatine deficiency syndromes: clinical aspects, treatment and pathophysiology. Subcell Biochem. 2007:46:149-66.

11. Salomons GS, van Dooren SJ, Verhoeven NM, Marsden D, Schwartz C, Ceci KM, et al. X-linked creatine transporter defect: an overview. J Inherit Metab Dis. 2003;26(2-3):309-18

12. Clark JF, Cecil KM. Diagnostic methods and recommendations for the cerebral creatine deficiency syndromes. Pediatr Res. 2015;77(3):398-405.

13. Clark AJ, Rosenberg EH, Almeida LS, Wood TC, Jakobs C, Stevenson RE, et al. X-linked creatine transporter (SLC6A8) mutations in about 1\% of males with mental retardation of unknown etiology. Hum Genet. 2006;119(6):604-10.

14. Betsalel OT, Rosenberg EH, Almeida LS, Kleefstra T, Schwartz CE, Valayannopoulos V, et al. Characterization of novel SLC6A8 variants with the use of splice-site analysis tools and implementation of a newly developed LOVD database. Eur J Hum Genet. 2011;19(1):56-63.
15. Yang Y, Muzny DM, Reid JG, Bainbridge MN, Willis A, Ward PA, et al. Clinical whole-exome sequencing for the diagnosis of mendelian disorders. N Engl J Med. 2013;369(16):1502-11.

16. Kuhlenbaumer G, Hullmann J, Appenzeller S. Novel genomic techniques open new avenues in the analysis of monogenic disorders. Hum Mutat. 2011;32(2):144-51.

17. Biesecker $L G$, Green $R C$. Diagnostic clinical genome and exome sequencing. N Engl J Med. 2014;370(25):2418-25.

18. Wang Q, Geng Q, Zhou Q, Luo F, Li P, Xie J. De novo paternal origin duplication of chromosome 11p15.5: report of two Chinese cases with Beckwith-Wiedemann syndrome. Mol Cytogenet. 2017:10:46.

19. Stelzer G, Plaschkes I, Oz-Levi D, Alkelai A, Olender T, Zimmerman S, et al. VarElect: the phenotype-based variation prioritizer of the GeneCards suite. BMC Genomics. 2016;17(Suppl 2):444.

20. Lion-Francois L, Cheillan D, Pitelet G, Acquaviva-Bourdain C, Bussy G, Cotton $F$, et al. High frequency of creatine deficiency syndromes in patients with unexplained mental retardation. Neurology. 2006:67(9):1713-4.

21. Betsalel OT, van de Kamp JM, Martinez-Munoz C, Rosenberg EH, de Brouwer AP, Pouwels PJ, et al. Detection of low-level somatic and germline mosaicism by denaturing high-performance liquid chromatography in a EURO-MRX family with SLC6A8 deficiency. Neurogenetics. 2008:9(3):183-90.

22. DesRoches $\mathrm{CL}$, Patel J, Wang P. Minassian B, Salomons GS, Marshall CR, et al. Estimated carrier frequency of creatine transporter deficiency in females in the general population using functional characterization of novel missense variants in the SLC6A8 gene. Gene. 2015;565(2):187-91.

23. Ardon O, Amat di San Filippo C, Salomons GS, Longo N. Creatine transporter deficiency in two half-brothers. Am J Med Genet A. 2010;152A(8):1979-83.

24. Kato H, Miyake F, Shimbo H, Ohya M, Sugawara H, Aida N, et al. Urine screening for patients with developmental disabilities detected a patient with creatine transporter deficiency due to a novel missense mutation in SLC6A8. Brain Dev. 2014;36(7):630-3.

25. deGrauw TJ, Salomons GS, Cecil KM, Chuck G, Newmeyer A, Schapiro MB, et al. Congenital creatine transporter deficiency. Neuropediatrics. 2002;33(5):232-8.

26. Kleefstra T, Rosenberg EH, Salomons GS, Stroink H, van Bokhoven H, Hamel $\mathrm{BC}$, et al. Progressive intestinal, neurological and psychiatric problems in two adult males with cerebral creatine deficiency caused by an SLC6A8 mutation. Clin Genet. 2005:68(4):379-81.

27. Jiddeke M, van de Kamp CJ, Michael K. Gibson,Gajja S. Salomons. New insights into creatine transporter deficiency:the importance of recycling creatine in the brain. J Inherit Metab Dis. 2013;36:155-6.

28. Heussinger N, Saake M, Mennecke A, Dorr HG, Trollmann R. Variable white matter atrophy and intellectual development in a family with X-linked Creatine transporter deficiency despite genotypic homogeneity. Pediatr Neurol. 2017:67:45-52.

29. Baroncelli L, Alessandri MG, Tola J, Putignano E, Migliore M, Amendola E, et al. A novel mouse model of creatine transporter deficiency. F1000Res. 2014:3:228.

30. Skelton MR, Schaefer TL, Graham DL, Degrauw TJ, Clark JF, Williams MT, et al. Creatine transporter (CrT; Slc6a8) knockout mice as a model of human CrT deficiency. PLoS One. 2011;6(1):e16187.

31. Nota B, Ndika JD, van de Kamp JM, Kanhai WA, van Dooren SJ, van de Wiel MA, et al. RNA sequencing of creatine transporter (SLC6A8) deficient fibroblasts reveals impairment of the extracellular matrix. Hum Mutat. 2014;35(9):1128-35.

Ready to submit your research? Choose BMC and benefit from:

- fast, convenient online submission

- thorough peer review by experienced researchers in your field

- rapid publication on acceptance

- support for research data, including large and complex data types

- gold Open Access which fosters wider collaboration and increased citations

- maximum visibility for your research: over $100 \mathrm{M}$ website views per year

At BMC, research is always in progress.

Learn more biomedcentral.com/submission 